\title{
AN EXPLORATION IN THE FIELD OF AGRICULTURAL KNOWLEDGE AND ITS BRIF STUDAY OF AGRICULTURE IN MADHYA PRADESH (Year 1999-2000 to Year 2009-2010)
}

\author{
Dr. Mukesh Mishra \\ Guest Faculty-Geography \\ Govt. Digree College \\ Batiyagarh, Damoh (MP), India
}

\begin{abstract}
In this paper, I have studied the agriculture of Madhya Pradesh and collected various agricultural related information by doing research related to it under this $I$ have told about the area of Madhya Pradesh its production and increase in yield in which $I$ have given an interval of five to five year, just as I have studied the area production and yield 1997, which year did I study from 1997 to the year 2002 to the year 2002 to the year 2007 to the year 2007 to the year 2012 as mentioned above and included in this paper and under it $I$ have Presented brief figures about soybean pedi wheat and gram which are shown in Table-1.

Similarly in my research paper I have found the difference of production of crops of Madhya Pradesh which I have shown in Table-2 and further I have shown in the type of land use and its presence in different districts and under Table-4 I have shown the field. (Agrawal, P.C.1960)

The types of soil is presented last and under Table-5 the types of soil as well as its receipt in different districts the less addressed but in table-6 I have explained the agricultural practices and in the Table together the difference of production of crops and finally I have described the high yielding crops under Table-8,So in relation to the improvement in the compact from we can say that this the research paper is a very brief study about the entire agriculture of Madhya Pradesh, so that we can easily understand what the state of agriculture in Madhya Pradesh what is more importance and how much income and development can the Madhya Pradesh government get from it.( Akhtar, R 1974).
\end{abstract}

Keywords - 1. Agricultural 2. Difference 3. production 4. Interval 5. Crops 6. Brief.

\section{INTODUCTION}

Madhya Pradesh is called the heartland of India which was formed on 1 November 1956 and it is situated between $21^{\circ} 6^{\prime}$ North latitude to $26^{\circ} 30^{\prime}$ North latitude to $74^{\circ} 91^{\prime}$ East longitude to $82^{\circ} 48^{\prime}$ East longitude in terms of latitudinal expansion and it has a population of 7 crore 26 lakh 26 thousand 809 inhabited and a subtropical climate.

I have studied the agriculture of Madhya Pradesh in this research paper and have also studied the entire agriculture closely under it India is an agricultural country, due to this Madhya Pradesh also has agriculture dominance here comes the first place under soyabeen agriculture a number of crops are seen but not only soyabeans many types of crops are cultivated here and their yield is also good. (Ayyar, N.P. 1969)

In which Madhya Pradesh government gets financially strong $12637.00 \quad\left(000^{\prime}\right.$ t. $)$ production was made under total crops as per year 1999 While there has been $3427.00\left(000^{\prime}\right.$ t. $)$ production under total pulse, 16064.00 (000't.) production has been done under total food grains, also the difference between the two has been determined by presenting the data. (Table-2).

I further explained the kind of land use pattern like forest, cultivation, uncultivated land, Total fallow land Total cropped area etc. figures are displayed in Table-3 under the Table- 4 the data for landholdings for the year 2001 and 2010 has been presented in which shoots of fields of less than 1 hectare and up to 10 hectares have been shot and in Table-5 all the friends of Madhya Pradesh and Their presence in various districts in indicated. (Ali Mohammad 1977)

Under Table-6 the cropping pattern of Madhya Pradesh is given such as figures of total creeds pulses all seeds etc. under total Kharif and rabi for the interval from 1999 to 2009 are displayed. Under Table-7 the difference of production of all the crosses in respect of different crops from year 1999 to year 2009 is derived and under Table-8 the difference of HYVS crops is presented in the context of your 1999 and year 2009.

Keeping in view the table it has been found that this entire research paper is based on the agriculture of Uttar Pradesh and the agriculture proof of all Madhya Pradesh is god addressing all the activities an research but sometimes the fields of agriculture there are fluctural such as if agricultural work is affected due to lack of time in time and the changes in agriculture due to this effect while you get to see your self so the exact cause should research a bit out in front of an agrarian state comes appearing in this paper finally it can be said that Madhya Pradesh.(Byress,T.J.1972)

Objectives:-

- Agriculture based on Madhya Pradesh

- Explanation of complete agricultural elements of Madhya Pradesh 


\section{International Journal of Engineering Applied Sciences and Technology, 2019 \\ Vol. 4, Issue 6, ISSN No. 2455-2143, Pages 214-224 \\ Published Online October 2019 in IJEAST (http://www.ijeast.com)}

- The complete description and status of agriculture based

- Important role of agricultural sector production,yields and crops.

- Status of agriculture thought intervals of different years.

- Complete information about the nature of agriculture in Madhya Pradesh.

- In corporation of complete facts related to agriculture.

\section{MATERIALS AND METHODS}

In preparing this research I have done a deep study related to it and collected data from primary books and reference books and prepared a research paper using explanatory descriptive method.

\section{RESULTS}

As a result in this research paper we have obtained the information of complete agriculture of Madhya Pradesh which is not easy to obtain as table-1 have come out that in the last five year in the year 1992 to2007 in Madhya Pradesh under the middle area of the year 1992 to 1997 at an interval of soyabean $(0.07 \%)$ Wheat $(3.33 \%)$ Paddy $(1.08 \%)$ gram $(3.25 \%)$ crops have changed respectively.If we look at table-1 as a whole then it was $19.01 \%$ production under 2007 and in the year 2007 itself the minimum production has been $-0.10 \%$ of the peri.

If we look at table -2 the highest reference to the difference in production of crops has been $16551.00(000$ 't.) in the year 2009-10 and under minimum total ailseed 720 (000't.) has been achieved in the year 1999-2000. The maximum absoulute change was 2388.00 (000't.) of the total qilseed and the difference between the minimum total percentage was 398.00 (000't.).
In table- 3 the Absdate change of the particulars with reference to the year 1999 and the year 2009 and has obtained a relieve change percentage In which the relative change of total fallow land has gone up to $8.86 \%$ and minimum $-18.54 \%$ and the maximum of total cropped area $9.92 \%$ and minimum $-3.10 \%$ has been achieved.( Dharandhar,K.P.1974)

Similarly after studying the land holdings within table-4 it has been found that between 2001 and year 2010 it was marginal (Below 1ha.) under the number of land and the maximum change has been $13.69 \%$ and minimum (10 ha.) $-55.75 \%$ change has come under the state.

The highest percentage of marginal (Below 1 ha.) has been obtained under Area 41.57 and minimum (above 10 ha.) data has been received under large. If the percentage change of entire crops is seen in table- 6 from the point of view of cropping pattern then the update has come down to minimum for example the highest percentage of Total Kharif pulses has been changed by only $0.38 \%$ and $-0.21 \%$ change has been achieved under minimum total Kharif cereals. (Dubey, R.S.1987)

The production of differential crops under table- 7 has a maximum change of $0.27 \%$ in total Kharif ailseeds in the interval from 1999 to 2009 and a minimum of $025 \%$ change in total Rabi pulses. The distribution of HYVS crops under table- 8 has obtained the highest $041 \%$ change in total pulses and the minimum $-0.57 \%$ change in fiber Kharif.

Acknowledgement:- This research paper written by me is perfection it is self made I have collected the data from the Agricultural statistics in Madhya Pradesh and through it I have Compiled the research paper with compiled honesty in this I calculating the data myself and keeping in mind the conclusions the data myself and keeping in mind the conclusions drawn from it. Have presented their ides in the from of research paper. ( Jain,S.C.1967)

Table-1

Growth of Area, Production and yield of Major crops in M.P. [At an interval of Five year] Percent Per year

\begin{tabular}{|c|c|c|c|c|c|}
\hline \multicolumn{2}{|c|}{ Five year plan } & $\begin{array}{l}\text { Soy } \\
\text { abee }\end{array}$ & Paddy & Wheat & Gram \\
\hline \multirow{3}{*}{$\begin{array}{l}1992- \\
1997\end{array}$} & $\begin{array}{l}\text { Area } \\
\text { ha.) }\end{array}$ & 0.07 & 1.08 & 3.33 & 3.25 \\
\hline & $\begin{array}{l}\text { Production } \\
\left(000^{\prime} \text { ha. }\right)\end{array}$ & 9.39 & 6.19 & 7.92 & 5.47 \\
\hline & $\begin{array}{l}\text { Yield (Q./ } \\
\text { ha.) }\end{array}$ & 1.64 & 0.35 & 4.77 & 2.48 \\
\hline \multirow{3}{*}{$\begin{array}{l}1997- \\
2002\end{array}$} & $\begin{array}{l}\text { Area } \quad\left(000^{\prime}\right. \\
\text { ha.) }\end{array}$ & 0.32 & 2.58 & -6.70 & -0.93 \\
\hline & $\begin{array}{l}\text { Production } \\
\left(000^{\prime} \text { ha. }\right)\end{array}$ & $\begin{array}{l}- \\
6.95 \\
\end{array}$ & 3.23 & -7.63 & -2.43 \\
\hline & $\begin{array}{l}\text { Yield } \\
\text { ha.) }\end{array}$ & $\begin{array}{l}- \\
7.26 \\
\end{array}$ & 1.97 & -0.04 & -1.69 \\
\hline \multirow{2}{*}{$\begin{array}{l}2002- \\
2007\end{array}$} & $\begin{array}{ll}\text { Area } & \left(000^{\prime}\right. \\
\text { ha. }) & \\
\end{array}$ & 3.15 & -0.01 & 3.76 & -0.04 \\
\hline & $\begin{array}{l}\text { Production } \\
\left(000^{\prime} \text { ha. }\right)\end{array}$ & $\begin{array}{l}10.6 \\
1\end{array}$ & 3.72 & 6.96 & 6.32 \\
\hline
\end{tabular}


International Journal of Engineering Applied Sciences and Technology, 2019

Vol. 4, Issue 6, ISSN No. 2455-2143, Pages 214-224

Published Online October 2019 in IJEAST (http://www.ijeast.com)

\begin{tabular}{|l|l|l|l|l|l|l|}
\hline & $\begin{array}{l}\text { Yield (Q./ } \\
\text { ha.) }\end{array}$ & 7.62 & 4.81 & 3.52 & 6.70 \\
\hline \multirow{2}{*}{$\begin{array}{l}2007- \\
2012\end{array}$} & $\begin{array}{l}\text { Area (000' } \\
\text { ha.) }\end{array}$ & $\begin{array}{l}\text { Production } \\
\text { (000' ha.) }\end{array}$ & 2.22 & -0.10 & 6.63 & 3.35 \\
\hline $\begin{array}{l}\text { Yield (Q./ } \\
\text { ha.) }\end{array}$ & 2.03 & 11.25 & 19.01 & 9.28 \\
\hline
\end{tabular}

Source:- Agricultural Statistics in M.P.

Table-2

Production of Different Total crops in M.P. (000' T.)

\begin{tabular}{|l|l|l|l|l|}
\hline Crops & $\begin{array}{l}\text { Years } \\
1999-2000\end{array}$ & $\begin{array}{l}\text { Year } \\
2009- \\
2010\end{array}$ & $\begin{array}{l}\text { Absolute } \\
\text { Change }\end{array}$ & $\begin{array}{l}\text { Relative } \\
\text { Change (\%) }\end{array}$ \\
\hline $\begin{array}{l}\text { Total } \\
\text { Cereals } \\
\text { Kharif) }\end{array}$ & 3842.00 & 4191.00 & 349.00 & 9.08 \\
\hline $\begin{array}{l}\text { Total } \\
\text { Cereals } \\
\text { (Rabi) }\end{array}$ & 8795.00 & 9331.00 & 536.00 & 6.09 \\
\hline Total & $\mathbf{1 2 6 3 7 . 0 0}$ & $\mathbf{1 3 5 2 2 . 0 0}$ & $\mathbf{8 8 5 . 0 0}$ & $\mathbf{7 . 0 0}$ \\
\hline $\begin{array}{l}\text { Total Pulse } \\
\text { (Kharif) }\end{array}$ & 442.00 & 459.00 & 17.00 & 3.85 \\
\hline $\begin{array}{l}\text { Total Pulse } \\
\text { (Rabi) }\end{array}$ & 2985.00 & 2570.00 & -415.00 & -13.90 \\
\hline Total & $\mathbf{3 4 2 7 . 0 0}$ & $\mathbf{3 0 2 9 . 0 0}$ & $\mathbf{- 3 9 8 . 0 0}$ & $\mathbf{- 1 1 . 6 1}$ \\
\hline $\begin{array}{l}\text { Total } \\
\text { Foodgrain } \\
\text { (Kharif) }\end{array}$ & 4284.00 & 4650.00 & 366.00 & 8.54 \\
\hline $\begin{array}{l}\text { Total } \\
\begin{array}{l}\text { Foodgrain } \\
\text { Rabi) }\end{array}\end{array}$ & 1178.00 & 11901.00 & 121.00 & 1.03 \\
\hline Total & $\mathbf{1 6 0 6 4 . 0 0}$ & $\mathbf{1 6 5 5 1 . 0 0}$ & $\mathbf{4 8 7 . 0 0}$ & $\mathbf{3 . 0 3}$ \\
\hline $\begin{array}{l}\text { Total } \\
\text { Oilseeds } \\
\text { Kharif) }\end{array}$ & 5025.00 & 7281.00 & 2256.00 & 44.90 \\
\hline $\begin{array}{l}\text { Total } \\
\text { Oilseeds } \\
\text { Rabi) }\end{array}$ & 720.00 & 852.00 & 132.00 & 18.33 \\
\hline Total & $\mathbf{5 7 4 5 . 0 0}$ & $\mathbf{8 1 3 3 . 0 0}$ & $\mathbf{2 3 8 8 . 0 0}$ & $\mathbf{4 1 . 5 7}$ \\
\hline
\end{tabular}

Source:- Agricultural Statistics in M.P.

FIGURE-1(Table-2)

Production(000'T.) Absolute Change of Different Total Crops in M.P. (Year 1999 To Year 2009)

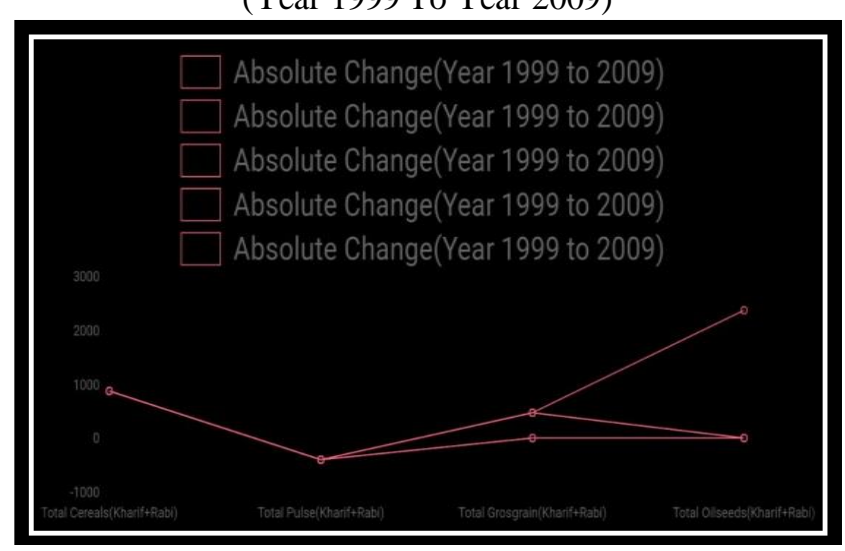

FIGURE-2(Table-2)

Production(000'T.) Relative Change of Different Total Crops in M.P. (Year 1999 To Year 2009)

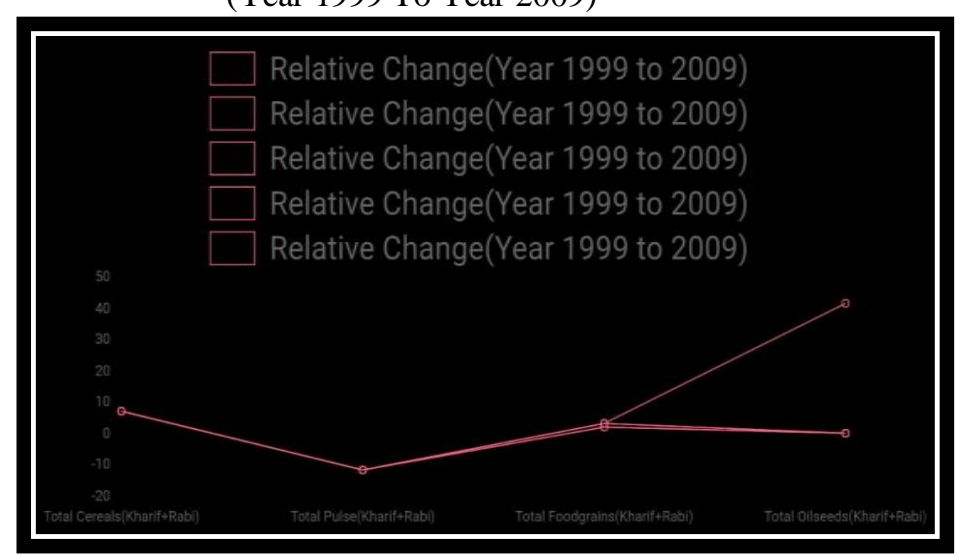


International Journal of Engineering Applied Sciences and Technology, 2019

Vol. 4, Issue 6, ISSN No. 2455-2143, Pages 214-224

Published Online October 2019 in IJEAST (http://www.ijeast.com)

Table-3

Land Use Pattern of M.P. (Lakh.ha.)

\begin{tabular}{|c|c|c|c|c|}
\hline $\begin{array}{l}\text { Partic } \\
\text { ulars }\end{array}$ & $\begin{array}{l}\text { Year } \\
1999- \\
2000\end{array}$ & $\begin{array}{l}\text { Year } \\
2009- \\
2010\end{array}$ & $\begin{array}{l}\text { Abs } \\
\text { olut } \\
\text { e } \\
\text { chan } \\
\text { ge }\end{array}$ & $\begin{array}{l}\text { Relati } \\
\text { ve } \\
\text { chang } \\
\text { e }(\%)\end{array}$ \\
\hline $\begin{array}{l}\text { Total } \\
\text { Geog } \\
\text { raphi } \\
\text { cal } \\
\text { area }\end{array}$ & $\begin{array}{l}307.50 \\
(100.00) \\
\%\end{array}$ & $\begin{array}{l}307.55 \\
(100.00 \\
) \%\end{array}$ & 0.05 & 0.02 \\
\hline $\begin{array}{l}\text { Fores } \\
\mathrm{t}\end{array}$ & $\begin{array}{l}86.11 \\
(28.00 \quad) \\
\%\end{array}$ & $\begin{array}{l}86.89 \\
(27.92) \\
\%\end{array}$ & 0.78 & 0.91 \\
\hline $\begin{array}{l}\text { Total } \\
\text { Not } \\
\text { availa } \\
\text { ble } \\
\text { for } \\
\text { cultiv } \\
\text { ation }\end{array}$ & $\begin{array}{l}32.00 \\
(10.41) \\
\%\end{array}$ & $\begin{array}{l}34.32 \\
(11.09) \\
\%\end{array}$ & 2.32 & 7.25 \\
\hline $\begin{array}{l}\text { Total } \\
\text { Uncu } \\
\text { ltivat } \\
\text { ed } \\
\text { land } \\
\text { exclu } \\
\text { ding } \\
\text { fallo } \\
\text { w } \\
\text { land }\end{array}$ & $\begin{array}{l}16.72 \\
(5.44) \%\end{array}$ & $\begin{array}{l}13.62 \\
(4.40) \\
\%\end{array}$ & $\begin{array}{l}- \\
3.10\end{array}$ & $\begin{array}{l}- \\
18.54\end{array}$ \\
\hline $\begin{array}{l}\text { Total } \\
\text { Fallo } \\
\mathrm{w} \\
\text { Land }\end{array}$ & $\begin{array}{l}10.61 \\
(3.45) \%\end{array}$ & $\begin{array}{l}11.55 \\
(3.91) \\
\%\end{array}$ & 0.94 & 8.86 \\
\hline $\begin{array}{l}\text { Total } \\
\text { Crop } \\
\text { ped } \\
\text { Area }\end{array}$ & $\begin{array}{l}204.19 \\
(66.40) \\
\%\end{array}$ & $\begin{array}{l}214.11 \\
(67.50) \\
\%\end{array}$ & 9.92 & 4.86 \\
\hline
\end{tabular}

Source:- Agricultural Statistics in M.P.

FIGURE-3(Table-3)

Absolute Change of Land Use Pattern in M.P. (Year 1999 To Year 2009)

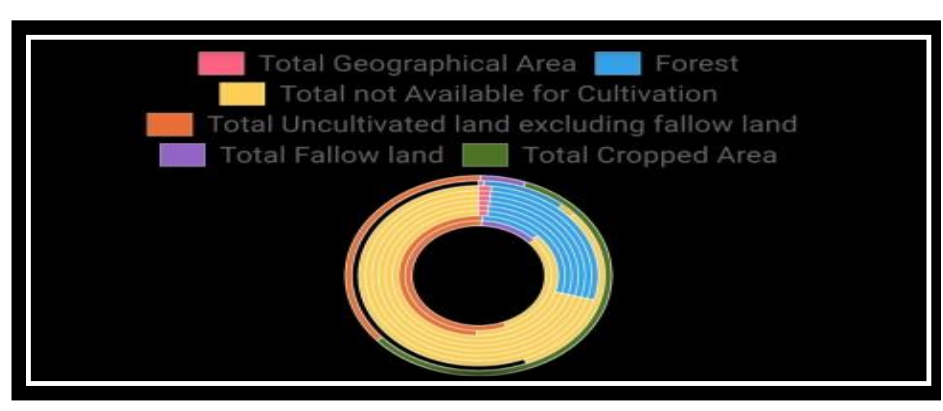

FIGURE-4(Table-3) Relative Change of Land Use Pattern in M.P.

(Year 1999 To Year 2009)

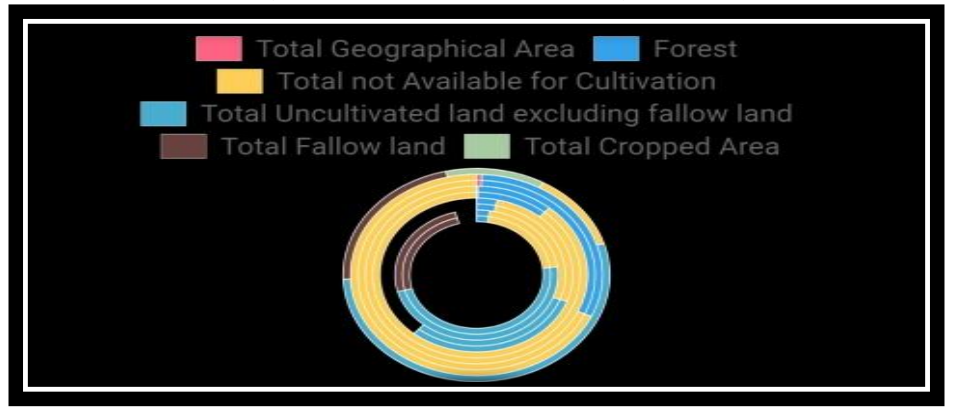

Table-4

Land Holdings in Madhya-Pradesh (Number in Lakh.)

\begin{tabular}{|c|c|c|c|c|c|}
\hline \multirow[b]{2}{*}{$\begin{array}{l}\text { Particula } \\
\text { rs }\end{array}$} & \multicolumn{2}{|c|}{$\begin{array}{l}\text { Year } \\
2001-2002\end{array}$} & \multicolumn{2}{|c|}{$\begin{array}{l}\text { Year } \\
2010-2011\end{array}$} & \multirow[b]{2}{*}{$\begin{array}{l}\% \\
\text { Cha } \\
\text { nge }\end{array}$} \\
\hline & $\begin{array}{l}\mathrm{Nu} \\
\mathrm{mb} \\
\text { er } \\
\text { (la } \\
\text { kh. } \\
\text { ) }\end{array}$ & $\%$ & $\begin{array}{l}\mathrm{Nu} \\
\mathrm{mb} \\
\mathrm{er} \\
\text { (la } \\
\mathrm{kh} . \\
\text { ) }\end{array}$ & $\%$ & \\
\hline $\begin{array}{l}\text { Marginal } \\
\text { (Below } \\
\text { 1ha.) } \\
\end{array}$ & $\begin{array}{l}28 . \\
38\end{array}$ & $\begin{array}{l}38 . \\
57\end{array}$ & $\begin{array}{l}38 . \\
51\end{array}$ & $\begin{array}{l}43.8 \\
5\end{array}$ & $\begin{array}{l}13.6 \\
9\end{array}$ \\
\hline $\begin{array}{l}\text { Small } \\
\text { (1 ha. to } \\
2 \text { ha.) }\end{array}$ & $\begin{array}{l}19 . \\
5\end{array}$ & $\begin{array}{l}26 . \\
51\end{array}$ & $\begin{array}{l}24 . \\
49\end{array}$ & $\begin{array}{l}27.6 \\
0\end{array}$ & 4.11 \\
\hline $\begin{array}{l}\text { Semi } \\
\text { Medium } \\
(2 \text { ha. To } \\
4 \text { ha.) }\end{array}$ & $\begin{array}{l}14 . \\
88\end{array}$ & $\begin{array}{l}20 . \\
22\end{array}$ & $\begin{array}{l}16 . \\
55\end{array}$ & $\begin{array}{l}18.6 \\
5\end{array}$ & - \\
\hline $\begin{array}{l}\text { Medium } \\
\text { ( } 4 \text { ha. To } \\
10 \text { ha.) }\end{array}$ & $\begin{array}{l}9.1 \\
6\end{array}$ & $\begin{array}{l}12 . \\
45\end{array}$ & $\begin{array}{l}7.8 \\
9\end{array}$ & 8.89 & $\begin{array}{l}- \\
28.5 \\
9 \\
\end{array}$ \\
\hline $\begin{array}{l}\text { Large } \\
\text { (Above } \\
10 \text { ha.) }\end{array}$ & $\begin{array}{l}1.6 \\
6\end{array}$ & $\begin{array}{l}2.2 \\
6\end{array}$ & $\begin{array}{l}0.8 \\
9\end{array}$ & 1.00 & $\begin{array}{l}- \\
55.7 \\
5 \\
\end{array}$ \\
\hline Total & $\begin{array}{l}73 . \\
59\end{array}$ & $\begin{array}{l}100 \\
.00\end{array}$ & $\begin{array}{l}88 . \\
73\end{array}$ & $\begin{array}{l}100 . \\
00\end{array}$ & \\
\hline
\end{tabular}

. Source:- Agricultural Statistics in M.P.

\section{FIGURE-5(Table-4)}

Parcentage Change Number(Lakh.) of Land Holdings in M.P.

(Year 2001 To Year 2010) 
International Journal of Engineering Applied Sciences and Technology, 2019

Vol. 4, Issue 6, ISSN No. 2455-2143, Pages 214-224

Published Online October 2019 in IJEAST (http://www.ijeast.com)

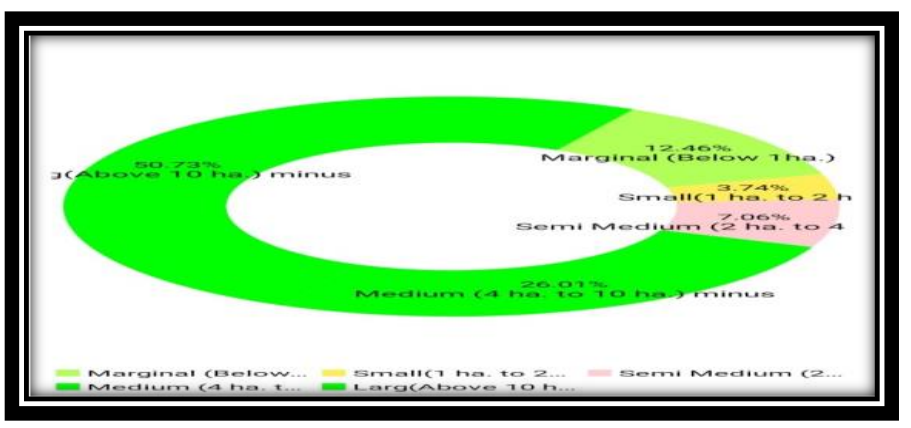

Table-5

Land Holdings in Madhya-Pradesh (Area in Lakh ha.)

\begin{tabular}{|c|c|c|c|c|}
\hline \multicolumn{2}{|c|}{$\begin{array}{l}\text { Year } \\
2001-2002\end{array}$} & \multicolumn{2}{|c|}{ Year 2010-2011 } & \multirow{2}{*}{$\begin{array}{l}\% \\
\text { Chan } \\
\text { ge }\end{array}$} \\
\hline $\begin{array}{l}\text { Area } \\
\text { (Lakh. } \\
\text { ha.) }\end{array}$ & $\%$ & $\begin{array}{l}\text { Area } \\
\text { (Lakh } \\
\text {.ha.) }\end{array}$ & $\%$ & \\
\hline 13.98 & 8.54 & 19.15 & $\begin{array}{l}12.0 \\
9\end{array}$ & 41.57 \\
\hline 28.28 & $\begin{array}{l}17.2 \\
8\end{array}$ & 34.66 & $\begin{array}{l}21.8 \\
9\end{array}$ & 26.68 \\
\hline 41.21 & $\begin{array}{l}25.1 \\
8\end{array}$ & 45.10 & $\begin{array}{l}28.4 \\
8\end{array}$ & 13.11 \\
\hline 54.47 & $\begin{array}{l}33.2 \\
8\end{array}$ & 45.45 & $\begin{array}{l}28.7 \\
0\end{array}$ & $\begin{array}{l}- \\
13.76\end{array}$ \\
\hline 25.75 & $\begin{array}{l}15.7 \\
3\end{array}$ & 14.00 & 8.84 & $-\overline{43.80}$ \\
\hline 163.69 & $\begin{array}{l}100 . \\
00\end{array}$ & $\begin{array}{l}158.3 \\
6\end{array}$ & $\begin{array}{l}100 . \\
00\end{array}$ & \\
\hline
\end{tabular}

Source:- Agricultural Statistics in M.P.

FIGURE-6(Table-5)

Percentage Change Area(Lakh.ha.)

of Land Holdings in M.P.

(Year 2001 To Year 2010)

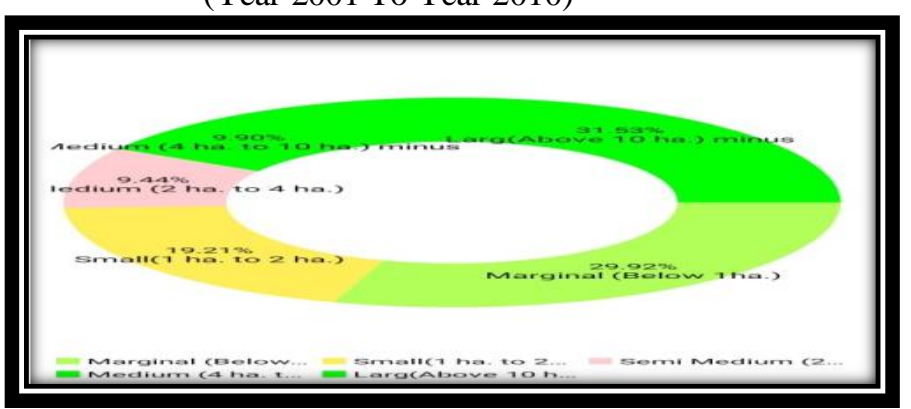

Table-6

Types and Sils found in different districts of Madhya Pradesh

\begin{tabular}{|c|c|}
\hline $\begin{array}{l}\text { Types } \\
\text { of Soil }\end{array}$ & Districts Covered \\
\hline $\begin{array}{l}\text { Alluvial } \\
\text { Soil }\end{array}$ & Bhind,Morena and Gwalior \\
\hline $\begin{array}{l}\text { Deep } \\
\text { Black } \\
\text { Soil } \\
\end{array}$ & Hoshangabad and Narsinghpur \\
\hline $\begin{array}{l}\text { Mediu } \\
\mathrm{m} \\
\text { Black }\end{array}$ & $\begin{array}{l}\text { Jabalpur,Sagar,Vidisha,Sehore, } \\
\text { Damoh,Guna,Bhopal,Raisan,Rajgarh,I } \\
\text { ndore,Dewas,Ujjain,Mandsour, }\end{array}$ \\
\hline
\end{tabular}

\begin{tabular}{|l|l|}
\hline Soil & $\begin{array}{l}\text { Shajapur,Ratlam,Dhar,Khargone and } \\
\text { Khandwa. }\end{array}$ \\
\hline $\begin{array}{l}\text { Shallow } \\
\text { Black } \\
\text { Soil }\end{array}$ & Betul,Chhindwara,and Seoni \\
\hline $\begin{array}{l}\text { Red and } \\
\text { black } \\
\text { Soil }\end{array}$ & $\begin{array}{l}\text { Shivpuri,Rewa,Satana,Panna,Sidhi,Chh } \\
\text { aterpur,Tikamgarh,Datia and some } \\
\text { parts of guna district. }\end{array}$ \\
\hline $\begin{array}{l}\text { Gravell } \\
\text { y Soil }\end{array}$ & Manda \\
\hline
\end{tabular}

Source:- Agricultural Statistics in MP

FIGURE-7(Table-6)

Types and Soils Food in Different Districts of M.P.

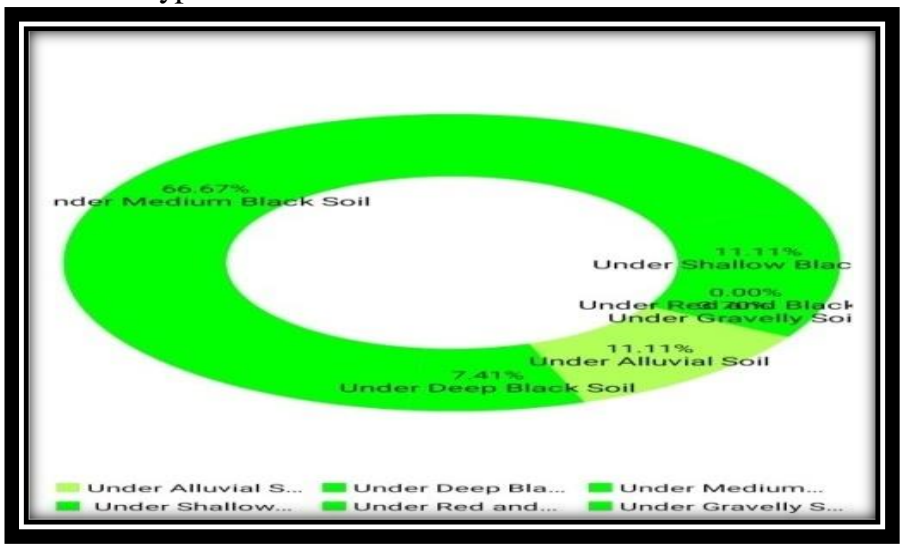

Table-7

Cropping Pattern in Madhya Pradesh

[Calculation of Percentage change from Gross Cropped Area and Percentage of year 1999 and year 2009] (000' ha.)

\begin{tabular}{|c|c|c|c|c|c|}
\hline Crops & $\begin{array}{l}\text { Year } \\
199 \\
9- \\
200 \\
0\end{array}$ & $\%$ & $\begin{array}{l}\text { Year } \\
2009- \\
2010\end{array}$ & $\%$ & $\begin{array}{l}\% \\
\text { Chang } \\
\text { e } \\
\text { (year } \\
1999 \\
\text { to year } \\
2009 \text { ) }\end{array}$ \\
\hline $\begin{array}{l}\text { Total } \\
\text { Kharif } \\
\text { Cereal } \\
\text { s }\end{array}$ & $\begin{array}{l}388 \\
4.00\end{array}$ & $\begin{array}{l}20 . \\
24\end{array}$ & $\begin{array}{l}3334 . \\
00\end{array}$ & $\begin{array}{l}15 . \\
92\end{array}$ & -0.21 \\
\hline $\begin{array}{l}\text { Total } \\
\text { Rabi } \\
\text { Cereal } \\
\text { s }\end{array}$ & $\begin{array}{l}476 \\
3.00\end{array}$ & $\begin{array}{l}24 . \\
82\end{array}$ & $\begin{array}{l}4727 . \\
00\end{array}$ & $\begin{array}{l}22 . \\
57\end{array}$ & -0.09 \\
\hline $\begin{array}{l}\text { Total } \\
\text { Cereal } \\
\text { S }\end{array}$ & $\begin{array}{l}864 \\
7.00\end{array}$ & $\begin{array}{l}45 . \\
05\end{array}$ & $\begin{array}{l}8061 . \\
00\end{array}$ & $\begin{array}{l}38 . \\
49\end{array}$ & -0.15 \\
\hline $\begin{array}{l}\text { Total } \\
\text { Kharif } \\
\text { Pulses }\end{array}$ & $\begin{array}{l}872 . \\
00\end{array}$ & $\begin{array}{l}4.5 \\
4\end{array}$ & $\begin{array}{l}1308 . \\
00\end{array}$ & $\begin{array}{l}6.2 \\
5\end{array}$ & $\begin{array}{l}0 . \\
38\end{array}$ \\
\hline $\begin{array}{l}\text { Total } \\
\text { Rabi }\end{array}$ & $\begin{array}{l}335 \\
4.00\end{array}$ & $\begin{array}{l}17 . \\
47\end{array}$ & $\begin{array}{l}3901 . \\
00\end{array}$ & $\begin{array}{l}18 . \\
63\end{array}$ & $\begin{array}{l}0 . \\
07\end{array}$ \\
\hline
\end{tabular}


International Journal of Engineering Applied Sciences and Technology, 2019

Vol. 4, Issue 6, ISSN No. 2455-2143, Pages 214-224

Published Online October 2019 in IJEAST (http://www.ijeast.com)

\begin{tabular}{|c|c|c|c|c|c|}
\hline \multicolumn{6}{|l|}{ Pulses } \\
\hline $\begin{array}{l}\text { Total } \\
\text { Pulses }\end{array}$ & $\begin{array}{l}422 \\
6.00\end{array}$ & $\begin{array}{l}22 . \\
02\end{array}$ & $\begin{array}{l}5209 . \\
00\end{array}$ & $\begin{array}{l}24 . \\
87\end{array}$ & $\begin{array}{l}0 . \\
13\end{array}$ \\
\hline $\begin{array}{l}\text { Total } \\
\text { Kahrif } \\
\text { Oilsee } \\
\text { ds }\end{array}$ & $\begin{array}{l}492 \\
6.00\end{array}$ & $\begin{array}{l}25 . \\
67\end{array}$ & $\begin{array}{l}4926 . \\
00\end{array}$ & $\begin{array}{l}23 . \\
52\end{array}$ & $\begin{array}{l}- \\
0 . \\
08\end{array}$ \\
\hline $\begin{array}{l}\text { Total } \\
\text { Rabi } \\
\text { oilseed }\end{array}$ & $\begin{array}{l}864 . \\
00\end{array}$ & $\begin{array}{l}4.5 \\
0\end{array}$ & $\begin{array}{l}823.0 \\
0\end{array}$ & $\begin{array}{l}3.9 \\
3\end{array}$ & $\begin{array}{l}- \\
0 . \\
13\end{array}$ \\
\hline $\begin{array}{l}\text { Total } \\
\text { oilssed }\end{array}$ & $\begin{array}{l}579 \\
0.00\end{array}$ & $\begin{array}{l}30 . \\
17\end{array}$ & $\begin{array}{l}5749 . \\
00\end{array}$ & $\begin{array}{l}27 . \\
45\end{array}$ & $\begin{array}{l}\overline{0} \\
0 . \\
09\end{array}$ \\
\hline
\end{tabular}

Source:- Agricultural Statistics in M.P

\section{FIGURE-8(Table-7)}

Cropping Pattern in M.P. Calculation of Percentage

Change from Gross Cropped Area(000'ha.) and

Percentage of Year 1999 and Year 2009

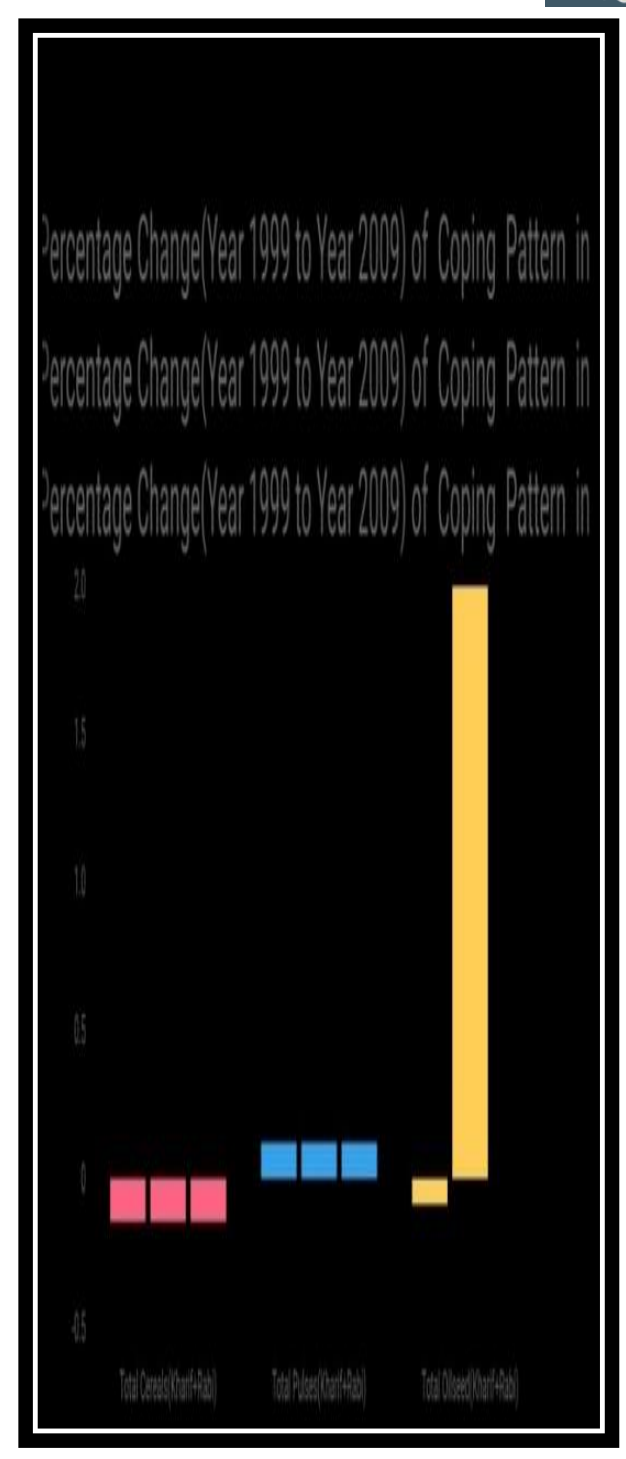

Table-8

Production of Different Crops in Madhya Pradesh [Calculation of percentage change from Gross cropped Area and percentage of year 1999 and year 2009]

Source:- Agricultural Statistics in M.P.

\begin{tabular}{|c|c|c|c|c|c|}
\hline Crops & $\begin{array}{l}\text { Year } \\
1999- \\
2000\end{array}$ & $\%$ & $\begin{array}{l}\text { Year } \\
2009- \\
2010\end{array}$ & $\%$ & $\begin{array}{l}\text { \% Change } \\
\text { (year 1999 } \\
\text { to 2009) }\end{array}$ \\
\hline $\begin{array}{l}\text { Total } \\
\text { Kharif } \\
\text { Cereals }\end{array}$ & 3842.00 & $\begin{array}{l}17.2 \\
9\end{array}$ & 4191.00 & $\begin{array}{l}16 . \\
50\end{array}$ & -0.05 \\
\hline $\begin{array}{l}\text { Total } \\
\text { Rabi } \\
\text { Cearea } \\
\text { ls }\end{array}$ & 8795.00 & $\begin{array}{l}39.5 \\
9\end{array}$ & 9331.00 & $\begin{array}{l}36 . \\
74\end{array}$ & -0.07 \\
\hline $\begin{array}{l}\text { Total } \\
\text { Cereals }\end{array}$ & 12637.00 & $\begin{array}{l}56.8 \\
8 \\
\end{array}$ & 13522.00 & 53.24 & -0.06 \\
\hline $\begin{array}{l}\text { Total } \\
\text { Kharif } \\
\text { Pulses }\end{array}$ & 442.00 & 1.99 & 459.00 & 1.81 & -0.09 \\
\hline $\begin{array}{l}\text { Total } \\
\text { Rabi }\end{array}$ & 2985.00 & $\begin{array}{l}13.4 \\
4\end{array}$ & 2570.00 & 10.12 & -0.25 \\
\hline
\end{tabular}


International Journal of Engineering Applied Sciences and Technology, 2019

Vol. 4, Issue 6, ISSN No. 2455-2143, Pages 214-224

Published Online October 2019 in IJEAST (http://www.ijeast.com)

\begin{tabular}{|c|c|c|c|c|c|}
\hline Pulses & & & & & \\
\hline $\begin{array}{l}\text { Total } \\
\text { Pulses } \\
\end{array}$ & 3427.00 & $\begin{array}{l}15.4 \\
3 \\
\end{array}$ & 3029.00 & 11.93 & -3.5 \\
\hline $\begin{array}{l}\text { Total } \\
\text { Kharif } \\
\text { Food grain }\end{array}$ & 4284.00 & $\begin{array}{l}19.2 \\
8\end{array}$ & 4650.00 & 18.31 & -0.05 \\
\hline $\begin{array}{l}\text { Total } \\
\text { Rabi } \\
\text { Food } \\
\text { grain }\end{array}$ & 11780.00 & $\begin{array}{l}53.0 \\
3\end{array}$ & 11901.00 & $\begin{array}{l}46 . \\
86\end{array}$ & -0.12 \\
\hline $\begin{array}{l}\text { Total } \\
\text { Food grain }\end{array}$ & 16064.00 & $\begin{array}{l}72.3 \\
1 \\
\end{array}$ & 16551.00 & $\begin{array}{l}65 . \\
16 \\
\end{array}$ & -0.09 \\
\hline $\begin{array}{l}\text { Total } \\
\text { Kharif } \\
\text { Oilseeds }\end{array}$ & 5025.00 & $\begin{array}{l}2 \\
2 \\
6 \\
6 \\
2\end{array}$ & 7281.00 & 28.67 & 0.27 \\
\hline $\begin{array}{l}\text { Total } \\
\text { Rabi } \\
\text { Oilseeds }\end{array}$ & 720.00 & 3.24 & 852.00 & 3.35 & 0.03 \\
\hline $\begin{array}{l}\text { Total } \\
\text { oilseeds }\end{array}$ & 5745.00 & $\begin{array}{l}25.8 \\
6\end{array}$ & 8133.00 & 32.02 & 0.24 \\
\hline $\begin{array}{l}\text { Total } \\
\text { Kharif }\end{array}$ & 9525 & $\begin{array}{l}42.8 \\
8 \\
\end{array}$ & 12450.00 & $\begin{array}{l}49 . \\
02\end{array}$ & 0.14 \\
\hline $\begin{array}{l}\text { Total } \\
\text { Rabi }\end{array}$ & 12690.00 & $\begin{array}{l}57.1 \\
2\end{array}$ & 12949.00 & 50.98 & -0.11 \\
\hline \multicolumn{6}{|c|}{$\begin{aligned} \text { Gross Cropped Area }= & 22215.00(000 ' \mathrm{t} .) \\
& \text { year }(1999-2000) \text { and } \\
& 25399.00(000 \text { ' t. }) \\
& \text { year } 2009-10\end{aligned}$} \\
\hline
\end{tabular}

FIGURE-9(Table-8)

Production of Different Crops

in M.P. Calucation of Percentage Change

from Gross Cropped Area $(000 ; \mathrm{T})$ and

Percentage of Year 1999 To Year 2009 
International Journal of Engineering Applied Sciences and Technology, 2019 Vol. 4, Issue 6, ISSN No. 2455-2143, Pages 214-224

Published Online October 2019 in IJEAST (http://www.ijeast.com)

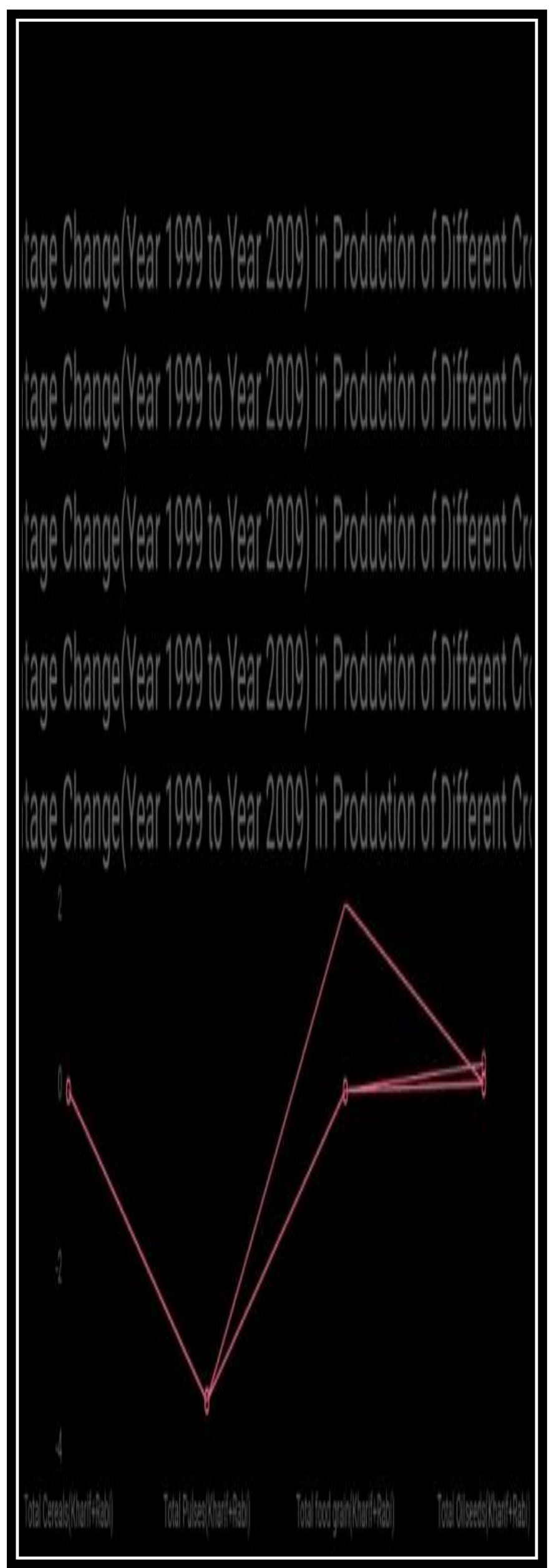


Published Online October 2019 in IJEAST (http://www.ijeast.com)

Table-9

Seed Distribution of HYVS crops in Madhya Pradesh (thousand q.)[Calculation of percentage change from Grand Total and percentage of year 1999 and year 2009]

\begin{tabular}{|c|c|c|c|c|c|c|}
\hline \multicolumn{2}{|c|}{ 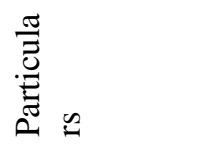 } & ঠ̋ & $b^{0}$ & పే & $8^{\circ}$ & 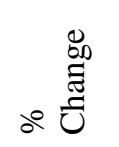 \\
\hline \multirow[b]{3}{*}{$\begin{array}{l}\frac{n}{\overparen{J}} \\
\stackrel{0}{0}\end{array}$} & $\begin{array}{l}\text { 节 } \\
\text { ป్ }\end{array}$ & 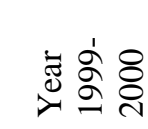 & $\delta^{\circ}$ & 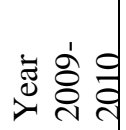 & $\delta^{\circ}$ & oீ \\
\hline & Rabi & 29.37 & $\begin{array}{l}3.4 \\
0\end{array}$ & $\begin{array}{l}13 \\
1 . \\
00\end{array}$ & $\begin{array}{l}3.5 \\
7\end{array}$ & 0.05 \\
\hline & Total & $\begin{array}{l}199.5 \\
6\end{array}$ & $\begin{array}{l}23 . \\
09\end{array}$ & $\begin{array}{l}60 \\
7 . \\
61 \\
\end{array}$ & $\begin{array}{l}16 . \\
54\end{array}$ & -0.28 \\
\hline \multirow[b]{3}{*}{$\begin{array}{l}\tilde{d} \\
\stackrel{0}{E} \\
\overrightarrow{2}\end{array}$} & $\begin{array}{l}\text { Khari } \\
\text { f }\end{array}$ & $\begin{array}{l}228.9 \\
36\end{array}$ & $\begin{array}{l}26 . \\
49\end{array}$ & $\begin{array}{l}73 \\
8 . \\
61\end{array}$ & $\begin{array}{l}20 . \\
11\end{array}$ & -0.24 \\
\hline & Rabi & 1.77 & $\begin{array}{l}0.2 \\
0\end{array}$ & $\begin{array}{l}13 \\
.2\end{array}$ & $\begin{array}{l}0.3 \\
6\end{array}$ & 0.8 \\
\hline & Total & 26.44 & $\begin{array}{l}3.0 \\
6\end{array}$ & $\begin{array}{l}15 \\
5 . \\
54\end{array}$ & $\begin{array}{l}4.2 \\
3\end{array}$ & 0.38 \\
\hline \multirow[b]{3}{*}{$\begin{array}{l}\frac{\tilde{u}}{\Delta} \\
\frac{w}{0} \\
0\end{array}$} & $\begin{array}{l}\text { Khari } \\
\text { f }\end{array}$ & 28.21 & $\begin{array}{l}3.2 \\
6\end{array}$ & $\begin{array}{l}16 \\
8 . \\
74\end{array}$ & $\begin{array}{l}4.5 \\
9\end{array}$ & 0.41 \\
\hline & Rabi & $\begin{array}{l}167.2 \\
3\end{array}$ & $\begin{array}{l}19 . \\
35\end{array}$ & $\begin{array}{l}91 \\
2 . \\
53\end{array}$ & $\begin{array}{l}24 . \\
84\end{array}$ & 0.28 \\
\hline & Total & 4.00 & $\begin{array}{l}0.4 \\
6\end{array}$ & $\begin{array}{l}10 \\
.1 \\
7\end{array}$ & $\begin{array}{l}0.2 \\
8\end{array}$ & -0.39 \\
\hline $\overrightarrow{\mathrm{d}}$ & $\begin{array}{l}\text { Khari } \\
\text { f }\end{array}$ & $\begin{array}{l}171.2 \\
3\end{array}$ & $\begin{array}{l}19 . \\
82\end{array}$ & $\begin{array}{l}92 \\
2 . \\
7\end{array}$ & $\begin{array}{l}25 . \\
12\end{array}$ & 0.27 \\
\hline$\frac{\bar{\theta}}{\bar{\theta}}$ & Rabi & 3.59 & $\begin{array}{l}0.4 \\
2\end{array}$ & $\begin{array}{l}6 . \\
77\end{array}$ & $\begin{array}{l}0.1 \\
8\end{array}$ & -0.57 \\
\hline \multirow{3}{*}{ 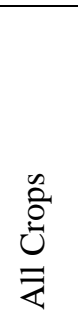 } & $\begin{array}{l}\text { Khari } \\
\mathrm{f}\end{array}$ & 0.07 & $\begin{array}{l}0.0 \\
08\end{array}$ & 0 & 0 & 0 \\
\hline & Rabi & $\begin{array}{l}201.9 \\
6\end{array}$ & $\begin{array}{l}23 . \\
37\end{array}$ & $\begin{array}{l}10 \\
63 \\
.5\end{array}$ & $\begin{array}{l}28 . \\
95\end{array}$ & 0.24 \\
\hline & Total & $\begin{array}{l}230.0 \\
7\end{array}$ & $\begin{array}{l}26 . \\
63\end{array}$ & $\begin{array}{l}77 \\
3 . \\
32\end{array}$ & $\begin{array}{l}19 . \\
96\end{array}$ & -0.25 \\
\hline
\end{tabular}




\section{International Journal of Engineering Applied Sciences and Technology, 2019 \\ Vol. 4, Issue 6, ISSN No. 2455-2143, Pages 214-224 \\ Published Online October 2019 in IJEAST (http://www.ijeast.com)}

\begin{tabular}{|c|c|c|c|c|}
\hline $\begin{array}{l}\text { Grand total year 1999- } \\
2006=(864.06 \text { th.q.) } \\
\text { and year } 2009-2010 \\
\text { (2673.64 th.q.) }\end{array}$ & & & & \\
\hline $\begin{array}{l}\text { The percentage change } \\
\text { of high variety crops } \\
\text { produced in Madhya } \\
\text { Pradesh is shown } \\
\text { under this table }\end{array}$ & $\begin{array}{l}432 \\
.03\end{array}$ & 50.00 & $\begin{array}{l}1836 . \\
82\end{array}$ & $\begin{array}{l}50 \\
.0 \\
0\end{array}$ \\
\hline
\end{tabular}

Source:- Agricultural Statistics in M.P.

\section{FIGURE-10(Table-9)}

Seeds Distribution of HYVS Crops

in M.P. Calculation of Percentage change

from Grand Total and percentage

(Year 1999 To Year 2009)

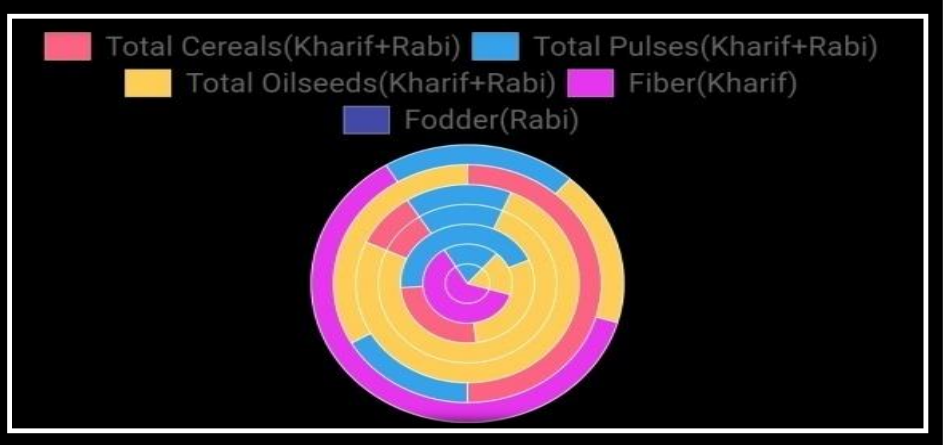

\section{DISCUSSION}

I think this research paper is a very good subject from the point of view of Madhya Pradesh because from this we have got the information of agriculture system of entire Madhya Pradesh, agricultural productivity and the close turn of all agriculture such as what percentage of the growth has been and how much area is there how much changed in the area, similarly all areas of production different land use patterns have been studied.

\section{CONCLUSION}

The entire paper concludes that most of the state of Madhya Pradesh is based on perfection agriculture and agriculture related industries are also run here on are helpful in the economy of Madhya Pradesh and Madhya Pradesh has also been awarded krishi karparn award in this area. Exept in jurisdictage only a normal percentage change is visible and mostly a percentage change the battle has gone to the end that is there is no change or development has not been done. In the year 2002 to year 2007 Growth has gone up to -0.04 (Area) than in the interval of year 1997 to year 20025 the percentage changed of yield has been $-1.69 \%$ therefore if there is no development in the agriculture of Madhya Pradesh it is neither complete nor undeveloped. Over all agriculture can be done in Madhya Pradesh for living.

\section{REFERENCES}

- P.C.Agrawal,"Measurement of Agriculture Efficiency in Bastar District,Asia",publishing House,pp,59,60.1960.

- R. Akhtar, "Agricultural Land use and Nutrition in Greater Himalayas",pp,74.1974.

- A.Mohammad, “ Studies in Agricultural Geography,Rajesh publication",New Delhi,pp,40,42.1978.

- A.Mohammad," Dynamics of Agricultural Development in India, concept publishing House, New Delhi,pp,70,71.1979.

- M.A.Ahluwalia," Rural poverty and Agricultural performance in India", Journal of Development studies,pp,80,81.1978.

- N.P.Ayyar,'The Agricultural Geography of the upper Narmada Basin",Unpublished PhD.Thesis,Sagar University,pp,94,97.1961.

- N.P. Ayyar and S. Shrivastav," Land use and Nutrition in Devas Basin M.P. India",pp,40,49.1968.

- N.P. Ayyar,"Crop Regions of Madhya Pradesh", Geographical Review of India,pp,20,27.1969

- A.Mohammad "Food and Nutrition in India", K.B. publication, New Delhi,pp,102,104.1977.

- S.Ansari,"Spatial Variation in Levels of socioeconomics Development of Ontario New perspective in Geography”, Allhabad,pp,104,110.1981.

- O.E.Beker,"Population Food supply and American Agriculture Geography cal Review”,pp,10,15.1928.

- ,G.S.Bholla and Y.K.Alagh,"performance of Indian Agriculture,sterling" Delhi,pp, 18,20.1979 publication, New

- G.Blyn,Measurement of growth Rate in Agriculture, Indian Journal of Agricultural Economics,pp,40,47.1967. 


\section{International Journal of Engineering Applied Sciences and Technology, 2019 \\ Vol. 4, Issue 6, ISSN No. 2455-2143, Pages 214-224 \\ Published Online October 2019 in IJEAST (http://www.ijeast.com)}

- B.M.Bhatia,"Poverty Agriculture and Economic Growth,Vikash"publication,New Delhi,pp,9,11.1977.

- M.C.Burk and M.A.Ezekiel,"Gricultural Development in Economic Growth",Cornell University press,pp,51,61.1970.

- D.D.Brown, “Agricultural Development in India's Districts” ,Harvard University Press,pp,78,91.1971.

- S.R. Basu," Development of water Resources of North-Eastern Region of India”,pp,71,72.1979.

- T.J. Byress, “The Dialectics of India's Green Revolution, south Asian Review”,pp,114,116.1972.

- T.S. Chouhan," Agricultural Geography",Jaipur,pp,47.1987.

- $\quad$ R.S. Chadha,"Growth rots in Agriculture Agricultural Situation in India",pp,78,79.1967.

- Chatterjee,Nondini ,'Methods for crop combination in regions of west Bengal Indian “,Journal of Land scap Systam,pp,40,41,1986.

- ,K.P.Dharandhar,"Soil Structure of Madhya Pradesh, The Indian Geographical Review",pp,30,31.1974.

- ,R.S.Dubey,"Agricultural Geography,Issues And Applications, Gian",

Delhi,pp,34,37.1987.

- Government of India ," The Food statistics of India,Deportment of Food",New Delhi,pp,40,70.1946.

- V.Hayami and V. Ruttan , "Agricultural Development,John”,Hopkins,Press,pp,29,30.1977.

- G..Hunter,"Agricultural development and Rural Poor,Concept", publication company,New Delhi,pp,19,20.1978.

- D.W.Harvey, "Theoretical concepts and the Analysis of Agricultural land use patterns in Geography",pp,10,11.1966.

- P.J.Iserma and H.O. Singer," Food Aid Its potential Disincentives to Agriculture Development Digest,pp,108,118.1977.

- Y.G. Johi,"The patterns of Agriculture in the Narmada River Basin,India",New Delhi,pp,40,41.1968.

- S.C. Jain,"More Food Through Controlled Agriculture", Kitab Mahal,Allhabad,pp,40,81.1967.

- ,R.K. Lekhi "Technological Revolution in Agriculture,classical”, publication,New Delhi,pp,40,42.1984.

- ,J. Mellor ,'The Economics of Agricultural Development",pp,37,38.1967.

- W.B.Morgan,"Agriculture in the Third world westview press", pp,37,39.1978.

- NCAER (1967): Cropping patterns in Madhya Pradesh,National council ofApplied Economic Research,New Delhi,pp,12,18.

- Stamp,L.D.(1931): The Land Utilisation Survey of Britain,Geographical Journal,pp,8,9.

- Stamp,L.D. (1958): The Measurement of Land Resources,Geographical Review,pp,7,8. 